\title{
Synthetic Breast Cancer Peptides-Tetanus Toxoid-Poly ICLC Vaccine
}

National Cancer Institute

\section{Source}

National Cancer Institute. Synthetic Breast Cancer Peptides-Tetanus Toxoid-Poly ICLC

Vaccine. NCI Thesaurus. Code C100099.

A cancer vaccine comprised of nine class I major histocompatibility complex (MHC)restricted breast cancer associated peptides, the tetanus toxoid helper peptide and the Toll-like receptor 3 (TLR3) agonist poly ICLC, with potential immunostimulatory and antineoplastic activities. The nine peptides derived from six cancer associated proteins are epidermal growth factor receptor 2 (HER2/neu), carcinoembryonic antigen (CEA) and four cancer/testis antigens (CTAs: MAGE-A1, -A3, -A10, and NY-ESO-1). Vaccination with this vaccine may elicit a specific cytotoxic T-lymphocyte $(C T L)$ response against cells overexpressing these tumor associated antigens (TAAs). As a nonspecific T-helper epitope, tetanus toxoid helper peptide binds to class II MHC and results in long-term immunopotentiation by increasing the helper T-cell response. Poly ICLC, the doublestranded RNA molecules of polyinosinic-polycytidylic acid stabilized with poly L-lysine in carboxymethylcellulose, binds to TLR3 and induces the release of cytokines which may help boost the immune response against the TAAs. 